\title{
Estudo da resistência cicatricial cutônea de ratos tratados com fototerapia a laser
}

\author{
Study of the strength of healing skin of rats treated with \\ phototherapy in laser
}

\author{
Kelsyanne de Castro Carvalho; ; Renata Amadei Nicolau²; Antônio Luis Martins Maia ${ }^{3}$; Paulo Roxo

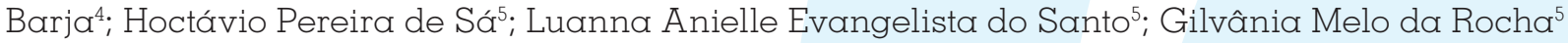 \\ ${ }^{1}$ Mestre em Engenharia Biomédica - Univap. São José dos Campos, SP - Brasil. Professora da Faculdade Santo Agostinho, Professora \\ do Centro de Ensino Unificado de Teresina. Teresina, PI - Brasil. \\ ${ }_{2}^{2}$ Professora Doutora em Engenharia Biomédica e Ciências Médica Básicas - Univap. São José dos Campos, SP - Brasil. \\ ${ }^{3}$ Mestre e Doutorando em Engenharia Biomédica - Univap. São José dos Campos, SP - Brasil. Professor - Faculdade Santo Agostinho, \\ Professor - Faculdade Integral Diferencial. Teresina, PI - Brasil. \\ ${ }^{4}$ Professor Doutor, Coordenador do PPG em Bioengenharia - IP\&D, Univap. São José dos Campos, SP - Brasil. \\ ${ }^{5}$ Acadêmico de Fisioterapia - Faculdade Santo Agostinho. Teresina, PI - Brasil. \\ Endereço para correspondência \\ Kelsyanne de Castro Carvalho \\ R. Ocílio Lago, 1257, Ininga \\ 64049-765 - Teresina - PI [Brasil] \\ kelsyanne@hotmail.com
}

\begin{abstract}
Resumo
Introdução: Terapia com laser tem sido amplamente estudada por seu efeito cicatrizante; contudo, poucos estudos foram desenvolvidos avaliando resistência à tensão pós-fototerapia. Objetivo: Analisar a tensão cicatricial em incisões cutâneas de ratos, após terapia laser (InGaAlP-670nm). Método: Vinte ratos Wistar, divididos em dois grupos (placebo/irradiado), sofreram incisão cutânea no dorso. O grupo irradiado recebeu dose de $6 \mathrm{~J} / \mathrm{cm}^{2}$ e no grupo placebo o equipamento manteve-se desligado. Após sacrifício, em três e sete dias pós-cirurgia, amostras de pele foram submetidas à análise tênsil-histológica. Resultados: Observou-se diferença significante na força de ruptura intergrupos, assim como tendência de diminuição na deformação máxima, porém sem expressividade estatística. Ocorreu redução do infiltrado inflamatório e aceleração de fibroplasia e angiogênese, compatíveis com elevação da síntese de colágeno na área das lesões no grupo irradiado. Conclusão: A terapia com laser de baixa intensidade aumenta a resistência tênsil da pele, melhorando a resposta reparacional, reduzindo riscos de deiscência e complicações pós-cirúrgicas.
\end{abstract}

Descritores: Cicatrização de feridas; Lasers; Pele.

\begin{abstract}
Introduction: Laser therapy has been widely studied for its healing; however, few studies have been developed to evaluate tensile strength after phototherapy. Objective: To analyze voltage scar skin incisions in rats after laser therapy (InGaAlP-670nm). Method: Twenty rats were divided into two groups (placebo/ irradiated), underwent skin incision on the back. The irradiated group received a dose of $6 \mathrm{~J} / \mathrm{cm}^{2}$ and in the placebo group the equipment remained off. After sacrifice, three and seven days post-surgery, skin samples were subjected to tensilehistological analysis. Results: It was observed significant difference in breaking strength between groups, decrease in maximum deflection (without statistical expression), reduction of the inflammatory infiltrate and acceleration of fibroplasia and angiogenesis, consistent with increased synthesis of collagen in the area of lesions in the irradiated group. Conclusion: Therapy with low-intensity laser increases the tensile strength of the skin, enhancing tissue repair response, reducing risks of dehiscence and postoperative complications.
\end{abstract}

Key words: Lasers; Skin; Wound healing. 


\section{Introdução}

A ferida constitui uma interrupção da continuidade de um tecido corpóreo, causada por qualquer tipo de trauma físico, químico, mecânico ou desencadeada por uma afecção clínica, que estimula a defesa do organismo lesionado iniciando o processo cicatricial. A cicatrização dessas lesões é um processo eficaz e dinâmico fisiologicamente, tendo como finalidade a recuperação da estrutura tissular para manter sua funcionalidade, mesmo com a perda tecidual que ocorre no início do processo lesão-reparação tecidual ${ }^{1}$.

$O$ processo de cicatrização tecidual envolve muitos eventos biológicos, tais como coagulação, inflamação, formação de tecido de granulação, contração do ferimento e remodelação tecidual. Após 24 horas do acometimento lesional, as células epiteliais migram da epiderme para invadir o coágulo. Em ferimentos de bordas bem-aproximadas, do tipo incisional, no decorrer de 48 horas uma camada contínua de células epiteliais recobre o ferimento. No terceiro ou quarto dia, o tecido de granulação se sobrepõe e inicia-se a deposição de colágeno. No primeiro mês, há uma proporcionalidade entre a resistência tênsil e a quantidade e qualidade do colágeno no ferimento ${ }^{2}$.

Os profissionais da área de saúde se deparam constantemente com desafios no tratamento de feridas não cicatrizadas, ou com dificuldade de cicatrização. A fisioterapia tem demonstrado atuar na prevenção e tratamento de tais feridas, usando recursos físicos como o laser, para acelerar o processo de cicatrização dos tecidos envolvidos ${ }^{3,4}$. A terapia com laser de baixa intensidade (TLBI) tem sido sugerida como opção terapêutica efetiva para modulação da dor e favorecimento do processo reparacional da cicatrização cutânea ${ }^{5}$.

Apesar de qualquer laser apresentar as características de monocromaticidade, coerência e colimação, os efeitos biológicos podem ser diferentes, dependendo do comprimento de onda, parâmetros de irradiação e características do tecido irradiado ${ }^{6}$.

Os lasers de baixa intensidade apresentam efeitos conhecidos como fotobiomoduladores os quais são importantes na cicatrização, no que se refere ao aumento da formação de tecido de granulação ${ }^{6,7}$, neovascularização ${ }^{8}$ e proliferação de fibroblastos $^{6}$, rápida epitelização $0^{8,9}$.

Interação eficiente do laser com o tecido tratado requer correspondência entre as características de um e outro (por exemplo, o tecido deve absorver bem a radiação no comprimento de onda emitido pelo laser). Quando radiação de baixa intensidade é usada, minimiza-se o efeito térmico e a energia absorvida, gerando essencialmente efeitos fotobiomoduladores ${ }^{10,11}$.

Apesar da existência de vários estudos sobre a fototerapia no processo cicatricial, a resistência da cicatriz é pouco abordada pela comunidade científica, embora seja extremamente importante na área clínica. Em decorrência disso, este estudo propôs a análise tensiométrica da pele, após terapia com laser, em cicatrizes incisionais no dorso de ratos.

\section{Materiais e método}

$\mathrm{O}$ projeto de pesquisa foi submetido à apreciação do Comitê de Ética e Pesquisa da Faculdade Integral Diferencial (Teresina, PI) e obteve aprovação, sob protocolo n $253 / 09$, seguindo os princípios éticos da experimentação animal, de acordo com as Normas Internacionais de Proteção aos Animais e do Colégio Brasileiro de Experimentação Animal (COBEA).

Foram estudados 20 ratos (Rattus norvegicus albinus) machos, da linhagem Wistar, pesando entre 200 a 250g, provenientes do biotério da Faculdade Santo Agostinho (Teresina, PI). Os animais foram distribuídos e acondicionados aleatoriamente em caixas específicas para ratos, mantidos desde o nascimento em um ciclo claro/escuro de 12/12 horas, com alimentação e água ad libitum (Tabela 1). 
Todos os animais receberam, por via subcutânea, um pré-tratamento com atropina (relaxante muscular), na dose de $0,04 \mathrm{~mL}$ para cada $100 \mathrm{~g}$ de peso corpóreo, aguardando-se 15 minutos para o procedimento anestésico ${ }^{12}$. Os animais foram submetidos à anestesia dissociativa com xilazina e quetamina na proporção de $1: 1$, na dose de $0,1 \mathrm{~mL}$ para cada $100 \mathrm{~g}$ do peso do animal, sendo depilados na região dorsal 24 horas antes do procedimento cirúrgico. Após a anestesia, realizou-se uma incisão longitudinalmente $(3 \mathrm{~cm})$ à pele da parte mediana do dorso, poupando músculos subjacentes. Em seguida, as bordas da ferida foram suturadas, com quatro pontos simples, sendo utilizado fio de nylon monofilamentar 4-0 $0^{13,14}$.

Tabela 1: Divisão de grupos experimentais

\begin{tabular}{c|c|c|c}
\hline $\begin{array}{c}\text { Grupo } \\
(\mathrm{n}=10)\end{array}$ & $\begin{array}{c}\text { Subgrupo } \\
(\mathrm{n}=5)\end{array}$ & Sacrifício & Tratamento \\
\hline \multirow{2}{*}{$\mathrm{A}$} & $\mathrm{A}_{3}$ & $3^{\circ} \mathrm{PO}$ & Placebo \\
\cline { 2 - 4 } & $\mathrm{A}_{7}$ & $7^{\circ} \mathrm{PO}$ & Placebo \\
\hline \multirow{2}{*}{$\mathrm{B}$} & $\mathrm{B}_{3}$ & $3^{\circ} \mathrm{PO}$ & Laser \\
\cline { 2 - 4 } & $\mathrm{B}_{7}$ & $7^{\circ} \mathrm{PO}$ & Laser \\
\hline
\end{tabular}

O grupo A (placebo) recebeu simulação de irradiação com equipamento de fototerapia desligado nos mesmos períodos do grupo tratado. O grupo B foi submetido a tratamento com irradiação a laser: i) nos 30 minutos posteriores à lesão, e ii), após 48 horas. A fototerapia foi realizada utilizando-se um laser de Fosfeto de Índio Gálio Alumínio (InGaAlP) de baixa intensidade (PHYSIOLUX DUAL, Bioset - Indústria de Tecnologia Eletrônica Ltda., Brasil). A irradiação foi realizada de forma pontual, transcutânea, perpendicularmente à pele do animal, com contado e localizada entre os pontos de sutura (Tabela 2) ${ }^{15}$.

Decorrido o experimento, procedeu-se ao sacrifício dos animais com aplicação de uma dose excessiva de anestesia ${ }^{13}$. A seguir, retirouse o fio de sutura e o fragmento de pele para análise tensiométrica e histológica ${ }^{14}$.

A resistência da cicatriz cutânea foi medida retirando-se um retalho de pele de $4 \times 2 \mathrm{~cm}$ transversal à cicatriz, e em sua parte média. Em
Tabela 2: Parâmetros de irradiação

\begin{tabular}{cc}
\hline \multicolumn{2}{c}{ Parâmetros do laser } \\
\hline Diodo & InGalP \\
\hline Densidade de energia & $6 \mathrm{~J} / \mathrm{cm} 2$ \\
\hline Potência & $16 \mathrm{~mW}$ \\
\hline Comprimento de onda & $670 \mathrm{~nm}$ \\
\hline Área do feixe & $0,09 \mathrm{~cm}^{2}$ \\
\hline Tempo & $33,7 \mathrm{~s}$ \\
\hline Números de pontos & 2 \\
\hline
\end{tabular}

seguida, as amostras foram alocadas em recipientes com solução de Ringer Lactato e encaminhadas imediatamente (tempo inferior a 60 minutos) para submissão ao teste de resistência à tensão no Laboratório de Ensaio Mecânico (Serviço Nacional de Aprendizagem Industrial - SENAI, Teresina - PI), por meio da Máquina de Ensaio DL20000 (Emic - Equipamentos e Sistemas de Ensaio Ltda, Brasil), com garras de pressão ajustáveis manualmente e sistema eletrônico de aquisição de dados com utilização do software Tesc, versão $1.01^{13}$.

A pele sofreu pinçamento por meio de um dispositivo de aço inoxidável com pressão gradual, observando-se a força de ruptura (FR) e a deformação máxima (DM). A FR corresponde ao maior valor de força necessário durante a tração da amostra, ou seja, o maior valor de resistência da amostra para que se atinja a ruptura; a DM dá o valor de deslocamento (deformação sofrida pela amostra) entre uma garra e outra do maquinário até a ruptura total do fragmento. A tração ocorreu com velocidade de $50 \mathrm{~mm} / \mathrm{min}$. e a força de tração foi constantemente mensurada automaticamente.

A porção distal da lesão $(1 \mathrm{~cm})$ foi fixada em formol a $10 \%$ e submetida a processamento histológico. De cada amostra foram obtidos oito cortes semisseriados $(5 \mu \mathrm{m})$, sendo quatro corados por Hematoxilina-Eosina, e quatro, com Tricrômico de Masson. A análise do número de fibroblastos, vasos sanguíneos e células inflamatórias foi realizada nos cortes corados com HematoxilinaEosina, e a análise da área do colágeno, nos cortes corados com Tricrômico de Masson. 
As lâminas histológicas foram submetidas à análise por microscopia óptica e captura de imagem digital, no laboratório de Fisiologia e Farmacologia do Instituto de Desenvolvimento e Pesquisa da Universidade do Vale do Paraíba. A captura das imagens foi efetuada por meio de uma câmara digital da marca Nikon ${ }^{\circledR}$, modelo Coolpix 5400, com resolução de 2592 X 1944 pixels e acoplada a um microscópio binocular, modelo Eclipse E200, da mesma marca, com objetivas planacromáticas. Para o acoplamento desses equipamentos usou-se um adaptador de câmera digital da marca Meiji Techno ${ }^{\circledR}$, modelo MA 151/40/52. As fotomicrografias foram obtidas em aumentos de 40 e 100 vezes e armazenadas no formato JPEG (Joint Photographic Experts Group).

Para a análise quantitativa das células, utilizou-se o programa ImageJ versão 1.43s. Através do plugin Cell Counter, realizou-se a contagem manual das células inflamatórias, fibroblastos e vasos sanguíneos. Com o plugin Threshold Colour, analisou-se a quantidade por área de colágeno. Todas as variáveis obtidas no estudo foram analisadas com o auxílio do programa GraphPad Prism ${ }^{\circledR}$, versão 2.00 . Os resultados foram submetidos à análise de variância (ANOVA) para múltiplas comparações, seguida do teste " $\mathrm{t}$ " Student para comparação entre os grupos placebo e laser, considerando um nível de significância de $5 \%(\mathrm{p}<0,05)$.

\section{Resultados}

Na observação macroscópica das lesões, não foi detectado nenhum sinal de infecção da ferida cirúrgica ou mesmo deiscência de sutura da pele.

\section{Anólise tensiométrica}

$\mathrm{Na}$ análise da tensão cicatricial dos retalhos cutâneos, no terceiro e sétimo dia pós-cirurgia, observou-se força de ruptura superior no grupo placebo no terceiro dia pós-cirurgia em relação ao grupo laser. No sétimo dia, após a cirurgia, a força de ruptura foi superior no grupo tratado com laser (Figura 1).

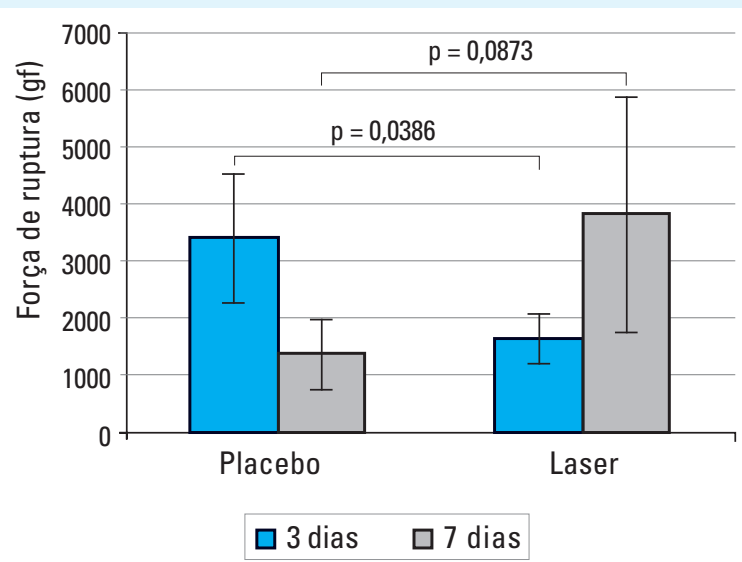

Figura l: Força de ruptura dos grupos placebo e laser no $3^{\circ}$ e $7^{\circ}$ dia pós-cirurgia. Valores expressos em média \pm desvio-padrão

Não foram observadas diferenças significativas na Deformação Máxima do retalho cutâneo entre os grupos placebo e laser, tanto no terceiro quanto no sétimo dia pós-cirurgia (Figura 2).

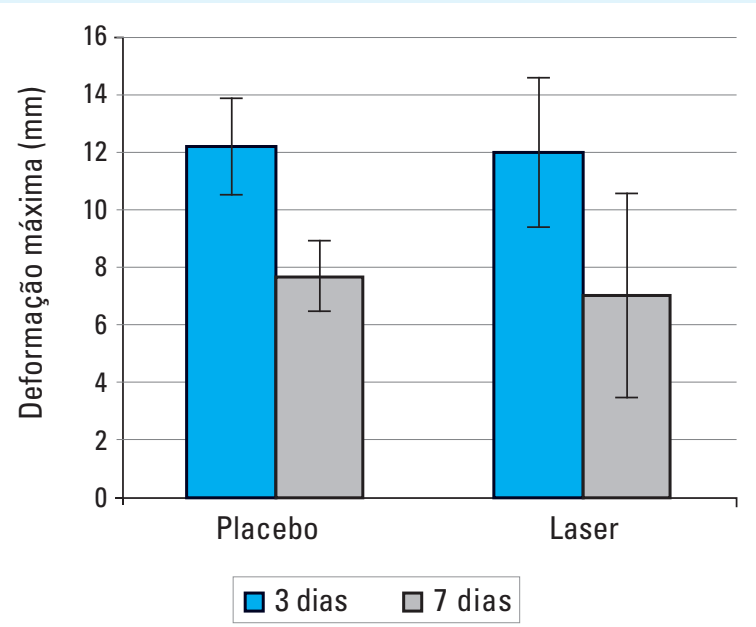

Figura 2: Gráfico comparativo das médias de Deformação Máxima dos diferentes grupos no terceiro e sétimo dia pós-cirurgia. Valores expressos em média \pm desvio-padrão

\section{Anólise histológica}

$\mathrm{Na}$ análise dos cortes histológicos verificou-se, no terceiro dia pós-cirurgia, um padrão 
histológico menos organizado que no sétimo dia pós-cirurgia em ambos os grupos (Figura 3).

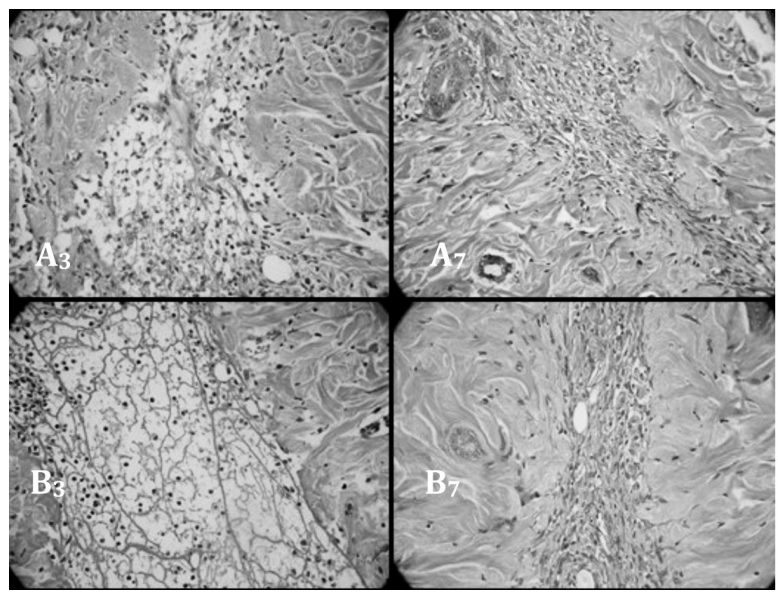

Figura 3: Fotomicrografias dos grupos experimentais nos diferentes momentos da reparação tecidual. Grupos placebo $\left(\mathrm{A}_{3} \mathrm{e}\right.$ $\left.A_{7}\right)$ e laser $\left(B_{3}\right.$ e $\left.B_{7}\right)$ no terceiro e sétimo dia pós-cirurgia

Em comparação ao grupo placebo (Figura 3. $\mathrm{A}_{3}$ ) observou-se menor concentração de células inflamatórias no grupo laser no terceiro dia. No grupo laser, pode-se observar maior presença de líquido em meio ao interstício no terceiro dia pós-cirurgia. Contudo, a presença de fibroblastos era mais evidente no grupo tratado com luz, nesse tempo experimental (Figura 3. $\mathrm{B}_{3}$ ).

No sétimo dia pós-cirurgia, verificou-se menor presença de células inflamatórias e de síntese no grupo laser. Nesse grupo, foi possível observar maior concentração de colágeno, com fibras bem organizadas, quando comparado ao grupo placebo, ambos no sétimo dia pós-cirurgia (Figuras 3. $A_{7}$ e $B_{7}$ ), indicando tecido mais

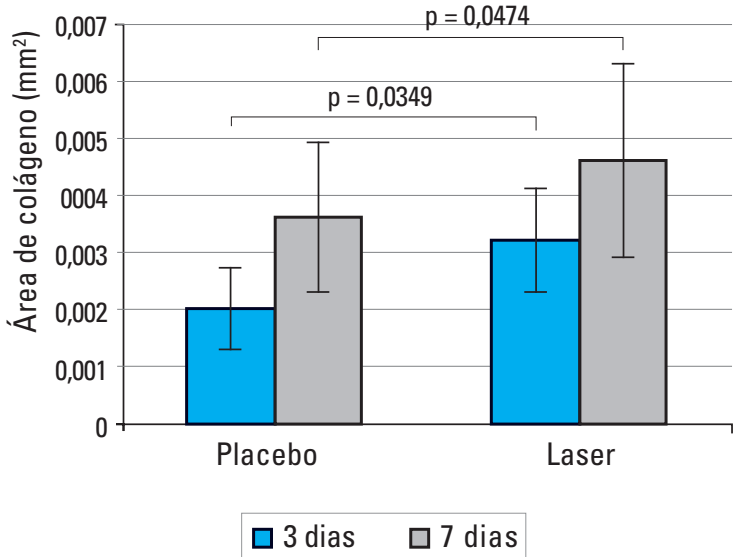

Figura 4: Área de cológeno no terceiro e sétimo dias pós-cirurgia. Valores expressos em média \pm desvio-padrão

maduro em comparação ao grupo placebo em mesmo período de reparo.

\section{Área do cológeno}

À medida que o reparo tecidual evolui os feixes de colágeno aumentam em número, tamanho e espessura proporcionando maior resistência à cicatriz (Figura 4).

A área de colágeno apresentou-se significativamente maior no grupo laser em relação ao grupo placebo, tanto no terceiro quanto no sétimo dia pós-cirurgia.

A cicatrização é um processo dinâmico no qual o tecido lesado é substituído por tecido conjuntivo vascularizado. Isso ocorre em fases do processo de reparação como inflamação, proliferação, síntese (fases iniciais) e remodelação (fase final). Na Tabela 3, podem ser observados os valores das células inflamatórias, fibroblastos e vasos, relacionados às fases iniciais do processo de reparo tecidual.

Tabela 3: Valores médios de células inflamatórias, fibroblatos e vasos no $3^{\circ}$ e $7^{\circ}$ dia pós-operatório

\begin{tabular}{|c|c|c|c|c|c|c|}
\hline \multirow{2}{*}{ Variáveis histológicas } & Placebo & Laser & \multirow{2}{*}{$P$} & Placebo & Laser & \multirow{2}{*}{$P$} \\
\hline & \multicolumn{2}{|c|}{$3^{\circ} \mathrm{dia}$} & & \multicolumn{2}{|c|}{$7^{\circ} \mathrm{dia}$} & \\
\hline Células inflamatórias & $217 \pm 25$ & $148 \pm 10$ & 0,0076 & $100 \pm 7$ & $70 \pm 13$ & 0,0029 \\
\hline Fibroblastos & $98 \pm 8$ & $114 \pm 13$ & 0,0447 & $108 \pm 9$ & $109 \pm 7$ & NS \\
\hline Vasos & $0,6 \pm 0,5$ & $3,0 \pm 1,6$ & 0,0161 & $1,4 \pm 1,1$ & $3,2 \pm 2,4$ & NS \\
\hline
\end{tabular}

Dados expressos em médias \pm desvio-padrão. $P=$ diferença significativa entre os grupos placebo e laser. NS = não significativo 
O número de células inflamatórias foi significativamente maior no grupo laser, tanto no terceiro quanto no sétimo dia pós-operatório. $\mathrm{O}$ número de fibroblastos e vasos foi significativamente maior no grupo tratado com laser no terceiro dia, quando comparado ao grupo placebo. Contudo, não foram observadas diferenças significativas entre os grupos no sétimo dia, tanto para o número de fibroblastos quanto de vasos sanguíneos.

\section{Discussão}

A proposição deste estudo foi observar o efeito da fototerapia a laser na cicatrização, após incisão em tecido cutâneo de ratos, enfatizando a resistência à tração dessa cicatriz e sua evolução histológica.

A TLBI é uma opção terapêutica para acelerar a cicatrização por sua ação bioestimuladora, analgésica e anti-inflamatória. Tem sido relatada a maior deposição de fibras colágenas, bem como a minimização da presença de células inflamatórias na zona de lesão, após terapia com laser ${ }^{16,17}$. Segundo estudos, esse tipo de terapia promove um efeito cicatricial por meio do aumento da atividade mitótica, número de fibroblastos, síntese de colágeno e neovascularização ${ }^{18,19}$.

A resistência de uma cicatriz operatória, medida pela tensão máxima, é um parâmetro bastante empregado na avaliação do processo de cicatrização ${ }^{21}$ o que justifica a escolha do método avaliativo proposto neste estudo para análise de tecido cutâneo tratado com equipamento de fototerapia, que tem por objetivo a otimização do reparo tecidual e o aumento da resistência cicatricial, principalmente na fase de síntese e remodelação.

No decorrer do processo cicatricial, iniciase a síntese do colágeno pelos fibroblastos, ao mesmo tempo em que ocorre o aumento da resistência tênsil da ferida. Nas primeiras duas semanas do processo reparacional, a resistência da cicatriz é diretamente proporcional à quantidade de colágeno depositada ${ }^{20,21,22}$. Comparando-se o grupo placebo com o tratado (laser), no terceiro dia pós-cirurgia, os resultados mostram que a média de força necessária para romper a cicatriz no grupo placebo alcança índices maiores que no tratado. No início do processo de reparação tecidual, o tecido cicatricial ainda é muito delicado e sua resistência é pequena e a resistência tênsil da cicatriz (tração) depende quase que exclusivamente da capacidade de sustentação das suas bordas ${ }^{13}$. Na análise histológica, observouse maior espaçamento no interstício, sugerindo presença de exsudato mais evidente no grupo tratado. Entretanto, o número de células inflamatórias foi menos evidente nesse grupo. Essa maior concentração de líquido no interstício e o número de vasos sanguíneos podem ter acarretado menor resistência à ruptura para o grupo laser. No entanto, essa reduzida resistência à tensão não comprometeu a deformação máxima do tecido. Após sete dias da lesão, o efeito de redução do processo inflamatório, associado ao estímulo celular (número de fibroblastos) e de síntese de colágeno acarretaram aumento da força de ruptura no grupo tratado em relação ao placebo. Segundo Nunes $\mathrm{Jr}^{23}$ a força de ruptura está relacionada com a resistência mecânica e com o conteúdo de fibras colágenas, enquanto a deformação máxima está relacionada com a elasticidade e, nesse caso, com o conteúdo de fibras elásticas. De acordo com Hawkins e Abrahamse ${ }^{24}$, os grupos tratados apresentam declínio brusco de células inflamatórias para ceder espaço aos fibroblastos, o que determina o recuo da fase inflamatória almejado na cicatrização de feridas incisionais ${ }^{25}$. Evidencia-se no sétimo dia maior número de fibroblastos no grupo tratado; com presença de fibras colágenas mais coesos e organizados, corroborando achados de Yasukawa et al. ${ }^{12}$. Esses dados confirmam estudo de Nascimento et al. ${ }^{26}$ em feridas incisionais, no qual observou a ação do laser HeNe (632,8 nm) sobre a aceleração da síntese de colágeno e diferenciação dos fibroblastos.

Estudos de Corazza et al. ${ }^{27}$ verificaram que a TLBI, com dose de $5 \mathrm{~J} / \mathrm{cm}^{2}$, estimula a angiogênese. A neoangiogênese é uma das caracte- 
rísticas histológicas mais importantes do tecido de granulação no processo de cicatrização cutânea ${ }^{19}$. No trabalho aqui apresentado, observouse um número de vasos superiores no grupo laser em relação ao grupo placebo no terceiro dia pós-cirurgia.

\section{Conclusão}

O tratamento com laser InGaAlP, no comprimento de onda 670nm e densidade de energia $6 \mathrm{~J} / \mathrm{cm}^{2}$, interferiu na cicatrização de peles de ratos incisionadas e suturadas, produzindo aumento na força de ruptura e diminuição da deformação máxima na análise tensiométrica, provavelmente pela síntese de colágeno mais pronunciada. A avaliação histológica evidenciou modulação do processo inflamatório pela TBLI, promovendo, assim, aceleração do reparo cicatricial.

\section{Referências}

1. Mandelbaum SH, Di Santis EP, Mandelbaum MHS. Cicatrização: conceitos atuais e recursos auxiliares Parte I. Anais Bras Dermatol. 2003;78(4):393-408.

2. Rodrigo SM, Cunha A, Pozza DH, Blaya DS, Moraes JF, Weber JBB et al. Analysis of the systemic effect of red and infrared laser therapy on wound repair. Photomed Laser Surg. 2009;27(6):929-35.

3. Hopkins JT, McLoda TA, Seegmiller JG, Baxter GD. Low-level laser therapy facilitates superficial wound healing in humans: a triple-blind, sham-controlled study. J Athl Train. 2004;39(3):223-9.

4. Benvindo RG, Braun G, Carvalho AR, Bertolini GRF. Efeitos da terapia fotodinâmica e de uma única aplicação de laser de baixa potência em bactérias in vitro. Fisioter Pesqui. 2008;15(1):53-7.

5. Silva FS, Araújo, AR, Chaves MEA, Pinotti M, Pereira, LFR. Diodo emissores de luz (LED) como terapia coadjuvante no tratamento de úlceras venosas - relato de caso. Revista Kinesia. 2009;1:18-27.
6. Dall Agnol MA, Nicolau RA, Liam CJ, Munin E. Comparative analysis of coherent light action (laser) versus non-coherent light (light-emitting diode) for tissue 6 repair in diabetic rats. Lasers Med Sci. 2009;24(6):909-16.

7. Bourguignon-Filho AM, Feitosa ACR, Beltrão GC, Pagnoncelli RM. Utilização do laser de baixa intensidade no processo de cicatrização tecidual. Revisão de literatura. Rev Port Estomatol Cir Maxilofac. 2005;46:37-43.

8. Rabelo SB, Villaverde AB, Nicolau RA, Salgado MAC, Melo MS, Pacheco MTT. Comparison between wound healing in induced diabetic and nondiabetic rats after low-level laser therapy. Photomed Laser Surg. 2006;24(4):474-9.

9. Medeiros JL, Nicolau RA, Nicola EM, Dos Santos JN, Pinheiro AL. Healing of surgical wounds made with lambda 970-nm diode laser associated or not with laser phototherapy (lambda $655 \mathrm{~nm}$ ) or polarized light (lambda 400-2000 nm). Photomed Laser Surg. 2009;26.

10. Rocha Júnior AM, Vieira BJ, Andrade LCF, Aarestrup FM. Effects of low-level laser therapy on the progress of wound healing in humans: the contribution of in vitro and in vivo experimental studies. J Vasc Bras. 2007;6(3):258-66.

11. Peng Q, Juzeniene A, Chen J, Svaasand L, Warloe T, Giercksky KE et al. Lasers in medicine. Rep Prog Phys. 2008;71:1-28.

12. Yasukawa A, Ohrui H, Koyama Y, Nagay M, Takakuda K. The effect of low reactive-level laser therapy (LLLT) with helium-neon laser on operative wound healing in rat model. J Vet Med Sci. 2007;68(8):799-806.

13. Alberti LR, Vasconcellos LS, Petroianu A. Resistência cicatricial cutânea sob efeito de hidrocortisona local ou sistêmica, em distintos períodos pós-operatórios. Einstein. 2008;6(3):269-73.

14. Veloso DFM, Petroianu A, Figueiredo JA, Alberti LR. Influência do hipoandrogenismo na resistência cicatricial cutânea em ratos. Einstein. 2009;7(1 Pt 1):1-4.

15. Arruda ERB, Rodrigues NC, Taciro C, Parizotto NA. Influência de diferentes comprimentos de onda da laserterapia de baixa intensidade na regeneração tendínea do rato após tenotomia. Rev Bras Fisioter. 2007;11(4):283-8.

16. Smith, KC. Laser (and LED) therapy is phototherapy. Letter to the editor. Photomed. Laser Surg. 2005;23(1):78-80. 
17. Leite SN, AndradeTAM, Minatel DG, Frade MAC. Fototerapia como estímulo à cicatrização de úlceras dorsais em ratos nutridos e desnutridos. Anais $21^{\circ}$ Congresso Brasileiro de Engenharia Biomédica. p.1090-3; 2008.

18. Pugliese LS, Medrado AP, Reis SRA, Andrade ZA. A influência da terapia a laser de baixa densidade de energia na biomodulação das fibras colágenas e elásticas. Pesqui Odontol Bras. 2003;17(4):307-13.

19. Balbino CA, Pereira LM, Curi R. Mecanismos envolvidos na cicatrização: uma revisão. Rev Bras Ciênc Farm. 2005;41(1):27-51.

20. Matayoshi S, Hanaoka BY, Osaka J, Tolosa EMC, Margarido N F. Comportamento biomecânico da pálpebra reconstruída. Rev Col Bras Cir. 2007;34(1):31-4.

21. Ehrlich HP, Keefer KA, Maish GO, Myers RL, Mackay DR. Vanadate ingestion increases the gain in wound breaking strength and leads to better organized collagen fibers in rats during healing. Plast Reconstr Surg. 2001;107(2):471-7.

22. Biondo-Simões MLP, Ioshii SO, Zazula AD, BiondoSimões R. O processo de cicatrização influenciado pelo hipotireoidismo e pelo envelhecimento. Estudo da cicatrização de anastomoses intestinais, em ratos. Acta Cir Bras. 2005;20(Supl1):113-9.
23. Nunes Jr JAT, Ribas-Filho JM, Malafaia O, Czeczko NG, Inácio CM Negrão AW, Lucena PLH, Moreira H, Wagenfuhr Jr J, Cruz JJ. Evaluation of the hydroalcoholic Schinus terebinthifolius Raddi (Aroeira) extract in the healing process of the alba linea in rats. Acta Cir Bras. 2006; (Supl 3)21:8-15.

24. Hawkins D, Abrahamse H. Phototherapy - a treatment modality for wound healing and pain relief. Afr J Biomed Res. 2007;10:99-109.

25. Demir H, Balay H, Kirnap M. A comparative study of the effects of electrical stimulation and laser treatment on experimental wound healing in rats. J Rehabil Res Dev. 2004;41(2):147-54.

26. Nascimento DG, Molena-Fernandes CA, Sartoretto JL, Bruschi LC, Cuman RKN, Silva FP. Efeitos da irradiação com o laser HeNe $632.8 \mathrm{~nm}$ sobre a cicatrização de feridas em ratos. Cien Cuid Saud. 2006;5(2):229-35.

27. Corazza AV, Jorge J, Kurachi C, Bagnato VS. Photobiomodulation on the angiogenesis of skin wounds in rats using different light sources. Photomed Laser Surg. 2007;25(2):102-6. 